\title{
Wetlands construídos empregados no tratamento de esgoto sob o contexto do saneamento de baixo carbono
}

Os processos de tratamento de esgotos sanitários emitem, em maior ou menor grau, gases de efeito estufa. No Brasil, a expressiva maioria da população de municípios de pequeno e médio portes que é atendida pelos serviços de esgotamento sanitário têm seus esgotos tratados por processos anaeróbios, cujo potencial de geração de metano pode ser até 80 vezes superior àquele associado aos processos aeróbios. Diante da realidade demográfica, geográfica e socioeconômica das cidades brasileiras, e sob o contexto de transição para uma economia de baixo carbono, os wetlands construídos (WC) despontam como importante alternativa tecnológica de tratamento de esgotos para municípios de até 20.000 habitantes. À luz dos guias de inventário de efeito estufa do Intergovernmental Panel on Climate Change - IPCC, o presente estudo objetivou analisar, em termos de redução de emissões de metano, o impacto da inserção dos WC na matriz brasileira de tecnologias de tratamento de esgotos. Considerando a projeção de universalização dos serviços de saneamento, as emissões de metano provenientes do tratamento de esgotos sanitários foram estimadas para três diferentes cenários de matriz tecnológica, variando o grau de participação dos WC. O cenário controle representa a manutenção das práticas de tratamento de esgoto conduzidas atualmente, e foi considerado como linha de base para o cálculo das reduções de emissão de metano nos demais cenários avaliados. Os resultados indicaram que, ao reduzir percentualmente a participação dos processos anaeróbios, a incorporação dos WC na matriz de tecnologias de saneamento no Brasil poderia reduzir as emissões totais de metano provenientes do tratamento de esgotos sanitários em mais de 10\%, valor que representa cerca de 867 milhões de toneladas de gás carbônico equivalente que deixam de ser emitidas anualmente. Ao serem transformadas em créditos de carbono, esta redução representa uma receita média de cerca de 35 milhões de dólares ao ano.

\section{Constructed wetlands applied for wastewater treatment from a low carbon sanitation perspective}

\begin{abstract}
Wastewater treatment plants (WWTP) can be a greater or lesser source of greenhouse gases depending on the technology used. In Brazil, most small and mediumsized municipalities served by sewage services adopt anaerobic processes, whose methane generation potential can be up to 80 times higher than that associated with aerobic processes. Considering demographic, geographic and socioeconomical aspects of Brazilian cities and facing the challenge of switching to a low-carbon economy, constructed wetlands (CW) emerges as an important process for wastewater treatment in municipalities with up to 20,000 inhabitants. Based on the Intergovernmental Panel on Climate Change (IPCC) guidelines for greenhouse gas (GHG) inventories, this study aimed at analyzing the potential impact on GHG emissions reduction by the entry of $\mathrm{CW}$ systems in the Brazilian wastewater treatment infrastructure. Assuming a universal sanitation coverage, methane emissions from municipal WWTP were estimated for three different infrastructure scenarios, each one with different participation of CW systems. The control scenario represents the maintenance of the wastewater treatment technologies currently adopted nationwide, and was considered as a baseline for methane emission reduction calculation in the other two scenarios. The results suggested that by switching the use of UASB reactors by CW systems, the methane emissions in the wastewater treatment infrastructure as a whole could be reduced by more than $10 \%$, a value that represents approximately 867 million tons of carbon dioxide equivalent that are no longer emitted annually. In terms of carbon credits, this reduction could represent an average revenue of US\$ 35 million per year.
\end{abstract}

Keywords: Low-carbon economy; Sanitation; Wastewater treatment; Constructed wetlands; Greenhouse gases.

Topic: Desenvolvimento, Sustentabilidade e Meio Ambiente

Reviewed anonymously in the process of blind peer.

Débora Carvalho Boratto (iD)

Universidade Federal de Minas Gerais, Brasil

http://lattes.cnpq.br/8976554532102607

http://orcid.org/0000-0001-8278-4416

debora-boratto@hotmail.com

André Baxter Barreto

Universidade Federal de Minas Gerais, Brasil

http://lattes.cnpq.br/3255065648145048

andre.barreto@wetlands.com.br

Pablo Heleno Sezerino (id

Universidade Federal de Santa Catarina, Brasil

http://lattes.cnpq.br/8752243933474803

http://orcid.org/0000-0002-2249-0878

pablo.sezerino@ufsc.br

DOI: 10.6008/CBPC2179-6858.2021.007.0035
Received: 02/07/2021

Approved: 28/07/2021

Cláudio Leite de Souza (D)

Universidade Federal de Minas Gerais, Brasil

http://lattes.cnpq.br/8283044406113169

http://orcid.org/0000-0003-3560-3488

claudio@desa.ufmg.br
Referencing this:

BORATTO, D. C.; BARRETO, A. B.; SEZERINO, P. H.; SOUZA, C. L.. Wetlands construídos empregados no tratamento de esgoto sob o contexto do saneamento de baixo carbono. Revista Ibero Americana de Ciências Ambientais, v.12, n.7, p.390-405, 2021. DOI: http://doi.org/10.6008/CBPC2179-6858.2021.007.0035 


\section{INTRODUÇÃO}

De acordo com o levantamento do Atlas Esgoto (ANA, 2020), apenas 1997 dos 5570 municípios brasileiros possuem estações de tratamento de esgoto sanitário (ETE), totalizando 3698 unidades (média de 1,85 ETE por município). Os principais processos utilizados, em termos do número de ETE, são os reatores anaeróbios (38\%), lagoas de estabilização (35\%), tanques sépticos (11\%) e lodos ativados (11\%). Outro levantamento realizado por Chernicharo et al. (2018) nas regiões Sul, Sudeste e Centro-Oeste do país, investigou 1667 ETE analisando seus portes e tipos de processos. Destas, 93\% (1551 ETE) atendem a populações menores que 100 mil habitantes, sendo que 57\% (945 ETE) atendem a populações menores que 10 mil habitantes. Os resultados demonstram ainda que, em municípios de até 100 mil habitantes, mais de $80 \%$ da população é atendida por reatores anaeróbios, lagoas de estabilização ou sistemas tanques sépticos seguidos de filtros anaeróbios.

Ambos os levantamentos retratam que, à exceção de algumas variantes das lagoas de estabilização (ex.: lagoas aeradas), a expressiva maioria da população de municípios de pequeno e médio porte que é atendida por ETE no Brasil têm seus esgotos tratados por processos anaeróbios. Além disso, estes levantamentos também apontam que a grande maioria das ETE atende populações inferiores a 100 mil habitantes, o que é coerente com a realidade demográfica brasileira, em que apenas 298 dos 5570 municípios possuem população acima de 100 mil habitantes.

Apesar das reconhecidas vantagens operacionais dos processos anaeróbios (baixos custos, simplicidade operacional e baixa geração de lodos), a degradação anaeróbia da matéria orgânica gera, dentre outros subprodutos, o metano $\left(\mathrm{CH}_{4}\right)$, gás de efeito estufa (GEE) com potencial de aquecimento global (GWP 100) 28 vezes superior ao gás carbônico (IPCC, 2006; IPCC, 2014a). Estudos realizados por Salomon et al. (2009), estimaram que a produção anual de metano proveniente do tratamento anaeróbio de esgoto doméstico no Brasil, à época, seria da ordem de $50 \mathrm{Gg} \mathrm{CH}_{4} \cdot \mathrm{ano}^{-1}(1 \mathrm{Gg}=1.000$ ton). Outro relatório do Sistema de Estimativas de Emissões de Gases de Efeito Estufa do Observatório do Clima (SEEG, 2019) aponta que, de 2000 a 2018, as emissões de GEE do setor de resíduos (que compreende o tratamento de efluentes e a disposição de resíduos sólidos), tiveram aumento de 95\%. Se, por um lado, este dado reflete a tendência de melhoria nas condições sanitárias do país, por outro, coloca em pauta a discussão sobre a atual matriz tecnológica para tratamento de esgotos, bem como a relevância de incorporar medidas de redução das emissões de carbono no setor. Neste mesmo relatório foi destacado que a universalização dos serviços de saneamento poderá promover aumento nas emissões de GEE, caso não sejam adotadas medidas contrárias às práticas de tratamento de esgoto conduzidas.

Nesse sentido, as principais alternativas tecnológicas para reduzir as emissões de GEE no tratamento de esgotos sanitários são o aumento da utilização de processos com baixo fator de emissão de metano (processos aeróbios) e a introdução de tecnologias para recuperação e/ou queima do metano/biogás nos reatores e digestores de lodo anaeróbios, especialmente para fins de aproveitamento energético (LIMA et al., 2014). 
Tendo em vista que cerca de $95 \%$ dos municípios brasileiros possuem população inferior a 100 mil habitantes (ANA, 2017b), a seleção de tecnologias de tratamento deve ser orientada pela simplicidade operacional e escala de viabilidade técnico-econômica. Processos aeróbios mais intensificados, como os sistemas de lodos ativados, tendem a ser inviáveis em municípios de pequeno e médio porte dada sua maior complexidade operacional, requisitos de mão de obra especializada e custo energético. Da mesma forma, o aproveitamento energético do biogás em reatores anaeróbios para produção de eletricidade é limitado pela escala de viabilidade econômica dos sistemas de conversão que, de maneira geral, está restrita às ETE de maior porte (acima de 100 mil habitantes) (CHERNICHARO et al., 2018).

Nesse contexto, os sistemas wetlands construídos despontam como uma excelente alternativa tecnológica para municípios com populações inferiores a 20.000 habitantes (75\% dos municípios brasileiros). Dentre as diversas tipificações de wetlands construídos, os sistemas de escoamento vertical descendente alimentados com esgoto bruto após tratamento preliminar (modelo francês - Figura 1) destacam-se como alternativa tecnológica para pequenos municípios, núcleos peri-urbanos e núcleos urbanos isolados. De forma resumida, estes filtros biológicos são construídos em tanques rasos escavados no solo $(1,0 \mathrm{~m}$ de profundidade), impermeabilizados com geomembrana de PEAD e preenchidos com materiais agregados com granulometrias especificadas em projeto. As redes de alimentação e drenagem são construídas com tubos de PVC ou PEAD. Sobre o maciço filtrante é plantada vegetação específica, adaptada a condições de alagamento temporário.

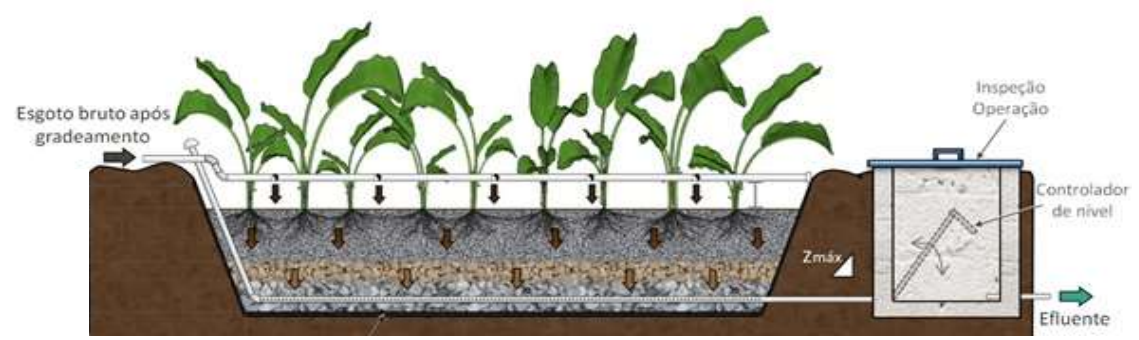

Figura 1: Esquema representativo em corte longitudinal de wetland construído de escoamento vertical - modelo Francês de primeiro estágio. Fonte: Adaptado de von Sperling et al. (2018).

Nestes sistemas, a alimentação ocorre sobre toda a superfície do leito de forma alternada e intermitente através de bomba elevatória ou sifão automático. Esta forma de alimentação permite a entrada de ar atmosférico por meio de convecção e subsequente difusão do ar pelo maciço filtrante e zona de raízes, permitindo a predominância de condições aeróbias e ausência de ambientes anaeróbios (VON SPERLING et al., 2018; KADLEC et al., 2009, STEFANAKIS et al., 2014). O fator de conversão do metano (Methane Conversion Factor - MCF), que representa o potencial de geração de $\mathrm{CH}_{4}$, em termos práticos para guiar os inventários de emissões, é até 80 vezes menor do que o MCF de processos anaeróbios (IPCC, 2019; IPCC, 2014b). O excelente desempenho da tecnologia, associado à sustentabilidade ambiental e ao elevado potencial de redução de custos operacionais envolvidos no tratamento de esgotos, vem aumentando o interesse pelo uso dessa tecnologia ao redor do mundo (STEFANAKIS, 2020). Apesar da crescente aplicação dos wetlands construídos no mundo, no Brasil esta tecnologia ainda é incipiente, havendo um grande potencial de ascensão. 
Frente às metas de expansão e universalização dos serviços de saneamento, o presente estudo avalia os potenciais impactos ambientais e financeiros, em termos de redução de emissão de GEE, em diferentes cenários de inserção da tecnologia wetlands construídos na matriz tecnológica brasileira para tratamento de esgotos sanitários.

\section{METODOLOGIA}

O presente estudo foi realizado a partir da estimativa e comparação das emissões de metano provenientes do tratamento de esgotos sanitários no Brasil considerando três diferentes cenários de matriz tecnológica, variando o grau de participação dos wetlands construídos.

Primeiramente, foram delimitadas as condições e premissas gerais do estudo, bem como os três cenários avaliados, que refletem diferentes percentuais de participação das tecnologias de tratamento de acordo com faixas populacionais. Em seguida, foram realizados os cálculos para (i) obtenção do fator de emissão de metano per capita para cada arranjo tecnológico avaliado, (ii) das estimativas de emissões anuais totais de metano para cada cenário e (iii) determinação da redução de emissão de metano com a implantação dos wetlands construídos, em termos quantitativos e monetários. Por fim, apresenta-se uma ferramenta para cálculo das reduções de emissão de GEE e obtenção de créditos de carbono com a utilização dos wetlands construídos em substituição a outras tecnologias.

\section{Delimitação das condições e premissas gerais do estudo}

O estudo considera a projeção de população urbana estimada a ser atendida por Estações de Tratamento de Esgoto (ETE) para o ano de 2035, para os 5570 municípios brasileiros. Estes dados, por sua vez, foram levantados com base no Censo do IBGE de 2010 e consideram a premissa da universalização do atendimento dos serviços de esgotamento sanitário. Os níveis de cobertura por rede coletora e tratamento por sistemas coletivos são considerados iguais ou superiores a 90\%, sendo complementados por soluções individuais. Ressalta-se que o levantamento não inclui os distritos e a população rural (ANA, 2017a; ANA, 2017b).

As estimativas consideram as emissões de metano $\left(\mathrm{CH}_{4}\right)$ provenientes do tratamento de esgoto sanitário pelas tecnologias de reatores anaeróbios, lagoas de estabilização, sistema de tanque séptico, sistemas aerados e wetlands construídos. As considerações acerca das variantes destes arranjos tecnológicos são: Reatores anaeróbios: considera-se que todas as ETE com reatores anaeróbios operam com queimadores de biogás (conversão de $\mathrm{CH}_{4}$ em $\mathrm{CO}_{2}$ ). O gás carbônico emitido não é contabilizado nos cálculos deste estudo, como indicado pelo IPCC. O tipo e as eficiências médias dos queimadores foram definidos de acordo com as práticas encontradas na realidade nacional de acordo com o porte da ETE, conforme segue (KAMINSKI et al., 2018): ETE pequeno porte: queimadores de gás abertos com 25\% de eficiência; ETE médio porte: queimadores de gás abertos com $50 \%$ de eficiência; ETE grande porte: queimadores de gás fechados (enclausurados) com 95\% de eficiência; Lagoas de estabilização: considera-se que 10\% são lagoas exclusivamente aeradas e os $90 \%$ restante compreendem arranjos que incluem lagoas anaeróbias e/ou 
facultativas, generalizados no estudo como sendo "sistemas australianos". Estes valores estão em concordância com os dados do Atlas Esgoto (ANA, 2020). As emissões provenientes das lagoas aeradas são contempladas no grupo dos sistemas aerados; Tanque Séptico: considera-se o arranjo utilizado para soluções coletivas que emprega a combinação sequencial entre tanque séptico e filtro anaeróbio. Para esse arranjo normalmente não há aplicação de queimadores, sendo os gases gerados dispersos na atmosfera;Lodos ativados/Outros: inclui as tecnologias de tratamento aeróbias aeradas, como lodos ativados e lagoas aeradas. As emissões de metano são provenientes principalmente dos decantadores e/ou de zonas do tratamento que apresentam eventuais condições anaeróbias; Sistemas wetlands construídos: considera-se a utilização da modalidade de wetland construído de escoamento vertical atuando em nível de tratamento secundário, com a utilização do primeiro estágio do wetland construído vertical modelo Francês empregado no tratamento de esgotos brutos após unidades preliminares de remoção de sólidos grosseiros.

Ressalta-se que, neste estudo, para o cálculo do gás carbônico equivalente $\left(\mathrm{CO}_{2 \text { eq }}\right)$ considera-se apenas as emissões de metano proveniente do processo de tratamento biológico, não computando: as emissões biogênicas de $\mathrm{CO}_{2}$, as emissões de óxido nitroso $\left(\mathrm{N}_{2} \mathrm{O}\right)$ e as emissões provenientes do consumo energético nas ETE (matriz energética local). Também não foram computadas as emissões de $\mathrm{CH}_{4}$ resultantes do lançamento de efluentes tratados nos corpos receptores e provenientes especificamente das etapas de tratamento e disposição dos lodos.

\section{Definição dos cenários}

Para todos os cenários, as análises foram realizadas considerando um agrupamento dos municípios de acordo com faixas populacionais da seguinte maneira: Pequeno Porte: subgrupo "a" $(<2.000$ hab.) e subgrupo "b" (entre 2.000 e 10.000 hab.); Médio Porte: subgrupo "a" (entre 2.000 e 20.000 hab.) e subgrupo "b" (entre 20.000 e 100.000 hab.); Grande Porte: > 100.000 hab.

Três cenários foram avaliados variando a participação dos wetlands construídos (percentual da população atendida pela tecnologia), sendo um cenário controle e outros dois cenários propositivos (cenário 01 e cenário 02).

Considerando a média nacional de 1,85 ETE por município com ETE e uma ampla faixa de aplicação dos wetlands, variando desde pequenos sistemas condominiais, comuns à realidade brasileira, até sistemas para vazões de até 20.000 equivalentes habitacionais ( 1 equivalente habitacional $=0,16 \mathrm{~m}^{3} / \mathrm{dia}$ ) (MASI et al., 2017), o uso desta tecnologia foi considerado aplicável para municípios de até 20.000 habitantes (ANA, 2020). Por este raciocínio, considera-se que uma cidade de 20.000 habitantes teria mais de uma ETE e poderia empregar esta tecnologia, uma vez que os portes de cada ETE seriam inferiores a 20.000 habitantes.

Os wetlands construídos foram inseridos nos dois cenários propositivos (cenários 01 e 02) em substituição aos reatores anaeróbios, lagoas de estabilização e fossas sépticas seguidas de filtros anaeróbios. A intenção foi substituir processos com maior emissão de GEE pelos wetlands construídos, para as faixas populacionais em que se aplicam, reduzindo a participação percentual dos processos anaeróbios na matriz tecnológica de saneamento. Os cenários mantêm inalterados os percentuais de atendimento por lodos 
ativados e outros arranjos tecnológicos não definidos, bem como o percentual de participação das tecnologias para municípios acima de 20.000 hab (médio porte " $b$ " e grande porte), em que os wetlands construídos não seriam aplicáveis para o tratamento de esgotos. A descrição de cada cenário será detalhada a seguir. A síntese dos três cenários encontra-se no Quadro 1.

\section{Cenário Controle}

O cenário controle representa a situação atual da matriz tecnológica brasileira, ou seja, a atual contribuição percentual das tecnologias em cada porte populacional. Para isso, considerou-se o panorama elaborado por Chernicharo et al. (2018), em que existe uma discriminação do percentual de atendimento por cada tecnologia nos diferentes portes populacionais nas regiões Sul, Sudeste e Centro Oeste do Brasil. Os valores de percentuais e portes populacionais levantados neste estudo para estas regiões foram considerados como representativos da realidade nacional e extrapolados para todos os municípios brasileiros para a construção do cenário.

O percentual de participação dos wetlands construídos foi considerado nulo. As emissões de GEE estimadas deste cenário são a linha de base para o cálculo das reduções de emissão de metano relativos aos outros dois cenários estudados, quando da entrada dos wetlands construídos na matriz tecnológica.

\section{Cenário 01}

Este cenário considera que a atual matriz tecnológica brasileira será moderadamente modificada, devido a um incremento conservador de participação da tecnologia wetlands construídos para os municípios de até $\mathbf{2 0 . 0 0 0}$ habitantes. Fatores que sustentam a proposição deste cenário incluem a crescente demanda por sistemas de tratamento de esgotos em pequenos e médios municípios, ampliação do know-how e domínio nacional sobre a tecnologia (fundamentado por pesquisas acadêmicas de longa duração), bem como da normatização e regulamentação da engenharia de aplicação desta tecnologia, o que tende a favorecer a sua disseminação e utilização em projetos de saneamento no país.

Para as faixas populacionais de pequeno porte e médio porte "a", considerou-se arbitrariamente uma redução percentual, em relação ao cenário controle, do atendimento por reatores anaeróbios, lagoas de estabilização e tanques sépticos seguidos de filtros anaeróbios de $10 \%$ para os dois primeiros arranjos e $25 \%$ para o último arranjo. O excedente passaria a ser atendido pela tecnologia wetlands construídos. Ou seja, $10 \%$ do número total de pessoas atendidas por Reatores Anaeróbios e Lagoas e $25 \%$ do número total de pessoas atendidas por Tanques Sépticos passam a ser atendidas pelos wetlands construídos.

\section{Cenário 02}

Para o cenário 02, o número de sistemas wetlands construídos implantados em outros países serviu como referência para estabelecer o percentual de participação na matriz tecnológica brasileira. Para as faixas populacionais de pequeno porte e médio porte "a", considerou-se uma redução percentual, em relação ao cenário controle, do atendimento por reatores anaeróbios, lagoas de estabilização e tanques sépticos de 25\% 
para os dois primeiros arranjos e 50\% para o último arranjo. 0 excedente passaria a ser atendido pela tecnologia wetlands construídos.

Dessa forma, os valores percentuais de atendimento por wetlands construídos encontrados para as faixas de população de pequeno porte passam a ser de $28,5 \%$, para o subgrupo "a", e $22,3 \%$ para o subgrupo "b". Estes valores estão em sintonia com a escala de utilização da tecnologia em países como a França, onde cerca de $22 \%$ de todas as ETE para até 10.000 habitantes possuem os wetlands construídos como alternativa tecnológica principal, e a Áustria, onde cerca de 30\% das novas estações de tratamento de esgotos de pequeno porte implementam essa tecnologia (FRANCE, 2018; LANGERGRABER et al., 2017).

Quadro 1: Matriz de tecnologias de tratamento de esgotos sanitários no Brasil nos cenários estudados.

\begin{tabular}{|c|c|c|c|c|c|c|c|c|c|}
\hline \multirow[b]{2}{*}{$\begin{array}{l}\text { CENÁRIOS } \\
\text { ESTUDADOS } 2035\end{array}$} & \multirow[b]{2}{*}{ FAIXA POPULACIONAL } & \multirow[b]{2}{*}{$\begin{array}{l}\mathbf{N}^{\circ} \\
\text { municípi } \\
\text { os }\end{array}$} & \multirow[b]{2}{*}{$\begin{array}{l}\mathbf{N}^{\circ} \\
\text { hab. }\end{array}$} & \multicolumn{6}{|c|}{ TECNOLOGIAS DE TRATAMENTO (\% de pessoas atendidas) } \\
\hline & & & & $\begin{array}{l}\text { Reatores } \\
\text { anaeróbios }\end{array}$ & $\begin{array}{l}\text { Lagoas de } \\
\text { estabilização }\end{array}$ & $\begin{array}{l}\text { Tanque } \\
\text { séptico }\end{array}$ & $\begin{array}{l}\text { Wetlands } \\
\text { construídos }\end{array}$ & $\begin{array}{l}\text { Lodos } \\
\text { ativados }\end{array}$ & $\begin{array}{l}\text { Out } \\
\text { ros }\end{array}$ \\
\hline \multirow{5}{*}{$\begin{array}{l}\text { Cenário Controle } \\
\text { matriz tecnológica } \\
\text { baseada no } \\
\text { panorama atual } \\
\text { (Chernicharo et } \\
\text { al., 2018) }\end{array}$} & $\begin{array}{l}\text { Pequeno porte "a" (<= } \\
\text { mil) }\end{array}$ & 645 & $\begin{array}{l}895.14 \\
5\end{array}$ & $25,0 \%$ & $41,0 \%$ & $24,0 \%$ & $0,0 \%$ & $7,0 \%$ & $\begin{array}{l}3,0 \\
\%\end{array}$ \\
\hline & $\begin{array}{l}\text { Pequeno porte "b" (2 } \\
\text { mil }<X<=10 \text { mil) }\end{array}$ & 2.636 & $\begin{array}{l}13.586 \\
501\end{array}$ & $37,0 \%$ & $48,0 \%$ & $2,0 \%$ & $0,0 \%$ & $11,0 \%$ & $\begin{array}{l}2,0 \\
\%\end{array}$ \\
\hline & $\begin{array}{l}\text { Médio porte "a" (10 mil } \\
<\mathrm{X}<=20 \text { mil) }\end{array}$ & 966 & $\begin{array}{l}13.749 . \\
082\end{array}$ & $52,0 \%$ & $29,0 \%$ & $0,0 \%$ & $0,0 \%$ & $18,0 \%$ & $\begin{array}{l}1,0 \\
\%\end{array}$ \\
\hline & $\begin{array}{l}\text { Médio porte "b" (20 mil } \\
<X<=100 \text { mil) }\end{array}$ & 1.016 & $\begin{array}{l}41.181 . \\
095\end{array}$ & $52,0 \%$ & $29,0 \%$ & $0,0 \%$ & $0,0 \%$ & $18,0 \%$ & $\begin{array}{l}1,0 \\
\%\end{array}$ \\
\hline & Grande porte (> $100 \mathrm{mil})$ & 307 & $\begin{array}{l}121.48 \\
7.125\end{array}$ & $22,0 \%$ & $7,0 \%$ & $0,0 \%$ & $0,0 \%$ & $44,0 \%$ & $\begin{array}{l}27,0 \\
\%\end{array}$ \\
\hline \multirow{5}{*}{$\begin{array}{l}\text { Cenário } 01 \\
\text { matriz tecnológica } \\
\text { considerando } \\
\text { incremento } \\
\text { conservador de } \\
\text { participação dos } \\
\text { wetlands }\end{array}$} & $\begin{array}{l}\text { Pequeno porte "a" (<= } \\
\text { mil) }\end{array}$ & 645 & $\begin{array}{l}895.14 \\
5\end{array}$ & $22,5 \%$ & $36,9 \%$ & $18,0 \%$ & $12,6 \%$ & $7,0 \%$ & $\begin{array}{l}3,0 \\
\%\end{array}$ \\
\hline & $\begin{array}{l}\text { Pequeno porte "b" (2 } \\
\text { mil }<X<=10 \text { mil) }\end{array}$ & 2.636 & $\begin{array}{l}13.586 . \\
501\end{array}$ & $33,3 \%$ & $43,2 \%$ & $1,5 \%$ & $9,0 \%$ & $11,0 \%$ & $\begin{array}{l}2,0 \\
\%\end{array}$ \\
\hline & $\begin{array}{l}\text { Médio porte "a" (10 mil } \\
<X<=20 \text { mil) }\end{array}$ & 966 & $\begin{array}{l}13.749 . \\
082\end{array}$ & $46,8 \%$ & $26,1 \%$ & $0,0 \%$ & $8,1 \%$ & $18,0 \%$ & $\begin{array}{l}1,0 \\
\%\end{array}$ \\
\hline & $\begin{array}{l}\text { Médio porte "b" (20 mil } \\
>\mathrm{X}<=100 \mathrm{mil})\end{array}$ & 1.016 & $\begin{array}{l}41.181 . \\
095\end{array}$ & $52,0 \%$ & $29,0 \%$ & $0,0 \%$ & $0,0 \%$ & $18,0 \%$ & $\begin{array}{l}1,0 \\
\%\end{array}$ \\
\hline & Grande porte (> $100 \mathrm{mil})$ & 307 & $\begin{array}{l}121.48 \\
7.125 \\
\end{array}$ & $22,0 \%$ & $7,0 \%$ & $0,0 \%$ & $0,0 \%$ & $44,0 \%$ & $\begin{array}{l}27,0 \\
\%\end{array}$ \\
\hline \multirow{5}{*}{$\begin{array}{l}\text { Cenário } 02 \\
\text { matriz tecnológica } \\
\text { considerando } \\
\text { participação dos } \\
\text { wetlands similar } \\
\text { ao que é } \\
\text { encontrado em } \\
\text { outros países }\end{array}$} & $\begin{array}{l}\text { Pequeno porte "a" (<= } 2 \\
\text { mil) }\end{array}$ & 645 & $\begin{array}{l}895.14 \\
5\end{array}$ & $18,8 \%$ & $30,8 \%$ & $12,0 \%$ & $28,5 \%$ & $7,0 \%$ & $\begin{array}{l}3,0 \\
\%\end{array}$ \\
\hline & $\begin{array}{l}\text { Pequeno porte "b" ( } 2 \\
\text { mil }<X<=10 \text { mil) }\end{array}$ & 2.636 & $\begin{array}{l}13.586 \\
501\end{array}$ & $27,8 \%$ & $36,0 \%$ & $1,0 \%$ & $22,3 \%$ & $11,0 \%$ & $\begin{array}{l}2,0 \\
\%\end{array}$ \\
\hline & $\begin{array}{l}\text { Médio porte "a" (10 mil } \\
<\mathrm{X}<=20 \text { mil) }\end{array}$ & 966 & $\begin{array}{l}13.749 . \\
082\end{array}$ & $39,0 \%$ & $21,8 \%$ & $0,0 \%$ & $20,3 \%$ & $18,0 \%$ & $\begin{array}{l}1,0 \\
\%\end{array}$ \\
\hline & $\begin{array}{l}\text { Médio porte "b" ( } 20 \text { mil } \\
>X<=100 \text { mil) }\end{array}$ & 1.016 & $\begin{array}{l}41.181 . \\
095\end{array}$ & $52,0 \%$ & $29,0 \%$ & $0,0 \%$ & $0,0 \%$ & $18,0 \%$ & $\begin{array}{l}1,0 \\
\%\end{array}$ \\
\hline & Grande porte (> $100 \mathrm{mil})$ & 307 & $\begin{array}{l}121.48 \\
7.125\end{array}$ & $22,0 \%$ & $7,0 \%$ & $0,0 \%$ & $0,0 \%$ & $44,0 \%$ & $\begin{array}{l}27,0 \\
\%\end{array}$ \\
\hline
\end{tabular}

\section{Cálculos}

Os cálculos relativos às emissões de metano foram realizados com base nos guias de inventário de gases de efeito estufa do Intergovernmental Panel on Climate Change - IPCC (IPCC, 2006; IPCC, 2014b; IPCC, 2019), utilizando metodologia básica (Tier 1 - valores padrão). As estimativas das reduções de emissão foram baseadas em conceitos gerais do mercado de créditos de carbono, como redução certificada de emissões (RCE) e venda de créditos de carbono. É importante ressaltar que o objetivo desses cálculos foi estimar a potencial redução nas emissões de GEE, não tendo sido utilizadas metodologias mais complexas de projetos de crédito de carbono. Os itens a seguir detalham as premissas, os cálculos e estimativas realizadas. 


\section{Premissas}

O MCF representa o potencial de geração de $\mathrm{CH}_{4}$ pelas tecnologias de tratamento de efluentes. Foram utilizados valores padrão do IPCC e/ou combinação desses valores para alguns arranjos tecnológicos (ver notas da Tabela 1). A redução de emissão pela queima do biogás nos reatores anaeróbios é contabilizada, posteriormente, no cálculo dos fatores de emissão.

Ressalta-se que a metodologia do IPCC não evidencia nos cálculos a eficiência de remoção de DBO. Todavia, pelos fundamentos da digestão anaeróbia, os fatores de emissão devem ser avaliados em termos de DBO removida, sendo necessário computar as eficiências típicas de remoção de DBO para cada tecnologia. Esta ponderação está alinhada com a abordagem adotada nos estudos de Noyola et al. (2016). A síntese dos valores utilizados é apresentada na Tabela 1.

Tabela 1: Valores de MCF e eficiências típicas de remoção de DBO para cada arranjo avaliado.

\begin{tabular}{|l|l|l|}
\hline Arranjo tecnológico & MCF & Eficiências típicas de remoção de DBO (\%) ${ }^{(2)}$ \\
\hline Reatores anaeróbios sem queima de gás & 0,8 & 70 \\
\hline Lagoas - sistema australiano $^{(1)}$ & 0,32 & 80 \\
\hline Tanque séptico + Filtro anaeróbio $^{(3)}$ & 0,5 & 80 \\
\hline Lagoas aeradas $^{(4)}$ & 0,03 & 80 \\
\hline Lodos ativados / Outros $^{(4)}$ & 0,03 & 95 \\
\hline Wetlands Sistema Francês & 0,01 & 85 \\
\hline
\end{tabular}

(1) MCF calculado pela média ponderada, em relação ao volume, dos MCF de lagoas facultativas e anaeróbias. Adota-se proporção de $20 \%$ lagoa anaeróbia e $80 \%$ lagoa facultativa (CETESB, 2006); (2) VON SPERLING (2018); CHERNICHARO (2016); VON SPERLING et al. (2018); (3) O IPCC não especifica o MCF para o arranjo tanque séptico + filtro anaeróbio. Por isso, adotou-se o valor de MCF relativo aos tanques sépticos; (4) Considera o MCF típico para estações de tratamento aeróbias centralizadas.

Os valores e referenciais adotados para as outras premissas utilizadas nos cálculos estão sintetizadas na Tabela 2. Ressalta-se que, devido a ampla variação do preço unitário da tonelada carbono, que é fortemente influenciada pelo país, tipo de projeto, ano e outros fatores, optou-se por considerar três possíveis valores (mínimo, médio e máximo).

Tabela 2: Premissas utilizadas para os cálculos.

\begin{tabular}{|c|c|c|c|}
\hline Variável & Unidade & Valor & Referência \\
\hline $\begin{array}{l}\text { Capacidade máxima de produção de } \\
\text { metano (Bo) }\end{array}$ & $\begin{array}{l}\mathrm{kg} \\
\text { DBOremovida }\end{array}$ & 0,6 & (IPCC, 2006) \\
\hline DBO per capita & g/hab.dia & 50 & von Sperling (2018) \\
\hline $\begin{array}{l}\text { Fator de correção para lançamentos } \\
\text { industriais (I) }\end{array}$ & - & 1,25 & (IPCC, 2006; IPCC, 2019) \\
\hline $\begin{array}{l}\text { Potencial para aquecimento global (PAG) } \\
\text { do metano }\end{array}$ & - & 28 & $\begin{array}{l}\text { Valor de referência do IPCC considerando horizonte de tempo de } \\
100 \text { anos (IPCC, 2014a) }\end{array}$ \\
\hline $\begin{array}{l}\text { Preço unitário da tonelada de carbono } \\
\text { (Punit) (médio) }\end{array}$ & USD & 40 & $\begin{array}{l}\text { Valor mínimo de referência recomendado para o ano de } 2020 \\
\text { (WBG, 2019) }\end{array}$ \\
\hline $\begin{array}{l}\text { Preço unitário da tonelada de carbono } \\
\left(P_{\text {unit }}\right) \text { (mínimo) }\end{array}$ & USD & 10 & $\begin{array}{l}\text { Valor médio utilizado pelas iniciativas de precificação de carbono } \\
\text { ativas (WBG, 2019) }\end{array}$ \\
\hline $\begin{array}{l}\text { Preço unitário da tonelada de carbono } \\
\left(P_{\text {unit }}\right) \text { (máximo) }\end{array}$ & USD & 80 & $\begin{array}{l}\text { Valor máximo de referência recomendado para o ano de } 2020 \\
\text { (WBG, 2019) }\end{array}$ \\
\hline
\end{tabular}

\section{Fatores de emissão de metano por arranjo e per capita}

O fator de emissão de metano para cada arranjo $\left(E F_{(k)}\right)$ foi calculado pela equação 1.

$$
E F_{(k)}=\left(M C F \times B_{0}\right)-R_{0} \quad \text { (Equação 1) }
$$

Onde: $E F$ = fator de emissão de metano $\left(\mathrm{kg} \mathrm{CH}_{4} / \mathrm{kg}\right.$ DBO removida $) ; B_{0}=$ capacidade máxima de produção de metano; $R_{0}=$ quantidade de metano recuperado (eficiência dos queimadores de biogás) ( $\mathrm{kg} \mathrm{CH}_{4} / \mathrm{kg} \mathrm{DBO}$ ); $\mathrm{k}=$ tipo de tecnologia/arranjo.

O fator de emissão de metano per capita para cada tecnologia $\left(E F_{\text {per capita }(k)}\right)$ foi então obtido pela 
equação 2. Este valor representa a estimativa de emissão anual de metano por habitante atendido por determinada tecnologia de tratamento de esgoto.

$$
E F_{\text {per capita }(k)}=E F_{(k)} \times D B O_{\text {per capita }} \times E_{(k)} \times I \times 0,001 \times 365
$$

Onde: $E F$ per capita $=$ fato de emissão de metano per capita $\left(\mathrm{kg} \mathrm{CH}_{4} / \mathrm{hab}\right.$.ano); $E F=$ fator de emissão de metano ( $\mathrm{kg}$ $\mathrm{CH}_{4} / \mathrm{kg}$ DBOremovida); $D B O_{\text {per capita }}=$ Demanda Bioquímica de Oxigênio per capita (g/hab.dia); $E=$ eficiência típica de remoção de DBO (\%); $I$ = fator de correção para lançamentos industriais; $\mathrm{k}$ = tipo de tecnologia/arranjo.

\section{Estimativas de emissões anuais de metano}

A emissão anual total de metano ( $\mathrm{g} \mathrm{CH}_{4} / \mathrm{ano}$ ) foi calculada pelo somatório das emissões estimadas de cada município. Estas, por sua vez, foram calculadas de acordo com a faixa populacional em que o município se enquadra e o seu respectivo fator de emissão per capita ponderado $\left(E F_{\text {pond }(i, c)}\right)$. $O$ fator de emissão per capita ponderado $\left(E F_{\text {pond }(i, c)}\right)$ representa a média ponderada dos $E F_{\text {per capita }(k)}$ considerando o percentual de pessoas atendidas por cada tecnologia em cada faixa populacional. Este procedimento foi feito para cada um dos três cenários e está representado pelas equações 3 e 4.

$$
E F_{\text {pond }(i, c)}=\text { Média ponderada }\left(E F_{\text {per capita }(k)} x W_{(k, i, c)}\right)
$$

Onde: $E F_{\text {pond }(i, c)}=$ fator de emissão per capita ponderado $\left(\mathrm{kg} \mathrm{CH}_{4} /\right.$ hab.ano); $\mathrm{i}$ = faixa populacional; $\mathrm{c}=$ cenário; $\mathrm{k}=$ tipo de tecnologia de tratamento; $E F_{\text {per capita }}=$ fato de emissão de metano per capita $\left(\mathrm{kg} \mathrm{CH}_{4} / \mathrm{hab}\right.$.ano); $W_{(k, i, c)}=$

$$
\text { Emissão } \mathrm{CH}_{4} \text { anual }(c)=\sum_{n=1}^{5570} \mathrm{EF}_{\text {pond }(i, c)} x \text { Pop }
$$
percentual de atendimento (\%).

(Equação 4)

Onde: Emissão $\mathrm{CH}_{4}$ anual $(c)=\mathrm{kg} C H 4 / a n o ; \mathrm{n}=$ municípios; $\mathrm{i}=$ faixa populacional; $\mathrm{c}=$ cenário; $E F_{\text {pond }(i, c)}=\mathrm{kg} \mathrm{CH} /$ hab.ano; $P o p=$ população do município (hab).

\section{Estimativas das reduções de emissão de metano}

A potencial redução de emissões de metano nos cenários 01 e 02 , em termos quantitativos e monetários, foi calculada tendo como base o cenário controle. Em termos quantitativos, efetuou-se a conversão das emissões totais de metano em cada cenário para toneladas de $\mathrm{CO}_{2}$ equivalentes e, em seguida, calculou-se a diferença entre as emissões totais de carbono dos cenários 01 e 02 em relação ao cenário controle. $\mathrm{O}$ valor encontrado representa as toneladas de carbono que deixariam de ser emitidas, caso as mudanças simuladas por aqueles cenários fossem adotadas (RCE). Os cálculos utilizaram as equações 5 e 6 .

$$
\text { Emissão } \mathrm{CO}_{2} \text { anual }(c)=\frac{\text { Emissão } C_{4} \text { anual }(c) x P A G}{1000}
$$

Onde: Emissão $\mathrm{CO}_{2}$ anual $(c)=$ ton $\mathrm{CO}_{2} / \mathrm{ano} ;$ Emissão $\mathrm{CH}_{4}$ anual $(c)=\mathrm{kg} \mathrm{CH} 4 / \mathrm{ano} ; \mathrm{c}=$ cenário; $\mathrm{PAG}=$ potencial

$$
R C E_{\left(c^{\prime}\right)}=\text { Emissão } \mathrm{CO}_{2} \text { anual }(c c)-\text { Emissão } \mathrm{CO}_{2} \text { anual }\left(c^{\prime}\right) \quad \text { (Equação 6) }
$$

Onde: $R C E$ = redução certificada de emissões (ton $\mathrm{CO}_{2} / \mathrm{ano}$ ) com base no cenário controle; $\mathrm{c}^{\prime}=$ cenário; $\mathrm{cc}=$ cenário controle; Emissão $\mathrm{CO}_{2}$ anual $=$ ton $\mathrm{CO}_{2} /$ ano.

Em termos monetários, estimou-se o valor referente à venda das RCE como crédito de carbono, segundo a equação 7 .

$$
\text { Créditos de carbono }\left(c^{\prime}\right)=R C E_{\left(c^{\prime}\right)} x P_{\text {unit }}
$$

Onde: $C r$ réditos de carbono = em USD; $R C E=$ redução certificada de emissões (ton $\mathrm{CO}_{2} / \mathrm{ano}$ ) com base no cenário controle; $\mathrm{c}^{\prime}$ = cenário; $P_{\text {unit }}=$ preço unitário tonelada de carbono (USD).

Por fim, para apresentação dos valores de redução de emissões per capita (e respectivo valor 
monetário) em função da utilização dos wetlands construídos como substituto a outras tecnologias de tratamento, utilizou-se as equações 8 e 9.

$$
\begin{aligned}
& R C E_{(W C)}=\frac{\left(E F_{\text {per capita }(k)}-E F_{\text {per capita }(k=w c)}\right) x P A G}{1000} \\
& C r e ́ d i t o s \text { de } \text { carbono }_{(W C)}=R C E_{(W C)} x P_{\text {unit }}
\end{aligned}
$$

Onde: $R C E_{(W C)}$ = redução de emissões per capita (ton $\mathrm{CO}_{2} / \mathrm{hab}$.ano) com a utilização dos wetlands construídos;

$E F_{\text {per capita }}=$ fator de emissão per capita $\left(\mathrm{kg} \mathrm{CH}_{4} / \mathrm{hab}\right.$.ano); $\mathrm{k}=$ tipo de tecnologia de tratamento (sendo $\mathrm{WC}=$ wetlands construídos); $P A G=$ potencial para aquecimento global do metano; $P_{\text {unit }}=$ preço unitário tonelada de carbono (USD).

\section{DISCUSSÃO TEÓRICA}

\section{Fatores de emissão de metano}

A Tabela 3 apresenta os fatores de emissão, em termos de carga orgânica removida (EF) e per capita $\left(E F_{\text {per capita }}\right)$, para cada arranjo tecnológico avaliado, calculados a partir das equações 1 e 2 . Ressalta-se que o

\begin{tabular}{|c|c|c|}
\hline Arranjo tecnológico & $\begin{array}{l}\mathrm{EF} \\
\left.\text { (kg CH} / 4 / \mathrm{kg} \mathrm{DBO}_{\text {remov }}\right)\end{array}$ & $\begin{array}{l}\text { EF } \quad \text { per } \\
\left(\mathrm{kg} \mathrm{CH}_{4} / \text { hab.ano) }\right.\end{array}$ \\
\hline Reatores anaeróbios sem queima de gás & 0,480 & 7,67 \\
\hline Reatores anaeróbios com $25 \%$ de queima & 0,360 & 5,75 \\
\hline Reatores anaeróbios com $\mathbf{5 0 \%}$ de queima & 0,240 & 3,83 \\
\hline Reatores anaeróbios com 95\% de queima & 0,024 & 0,38 \\
\hline Lagoas - sistema australiano & 0,192 & 3,50 \\
\hline Tanque séptico + Filtro anaeróbio & 0,300 & 5,48 \\
\hline Lagoas aeradas & 0,018 & 0,33 \\
\hline Lodos ativados / Outros & 0,018 & 0,39 \\
\hline Wetlands construídos - Sistema Francês & 0,006 & 0,12 \\
\hline
\end{tabular}
cálculo do $\mathrm{EF}_{\text {per capita }}$ considera as eficiências típicas de remoção de DBO.

Tabela 3: Fatores de emissão de metano referentes aos arranjos tecnológicos estudados.

Os wetlands construídos apresentaram o menor fator de emissão, já que o processo de tratamento destes sistemas engloba mecanismos predominantemente aeróbios. $\mathrm{O}$ arranjo dos reatores anaeróbios sem queima de gás, apresentou o maior valor de EF, cerca de 80 vezes maior do que aquele encontrado para os

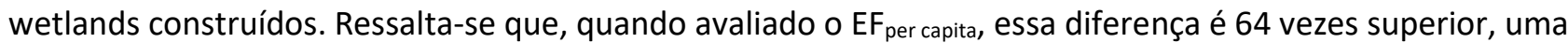
vez que os wetlands construídos apresentam maior eficiência de remoção de DBO (85\%) do que os reatores anaeróbios (70\%).

Outras tecnologias de tratamento convencionais predominantemente aeróbias, como as lagoas aeradas e lodos ativados, se bem operadas, possuem fator de emissão de 0,018 $\mathrm{kg} \mathrm{CH}_{4} / \mathrm{kgDBO}_{\text {remov. }}$, ligeiramente superior ao que é encontrado para os wetlands construídos. As metodologias do IPCC não justificam essa diferença, mas isso pode estar associado à formação de zonas anaeróbias, por exemplo, nos decantadores primários e/ou secundários.

\section{Considerações sobre a metodologia e os valores obtidos}

O valor geral para fator de emissão dos reatores anaeróbios $(0,480 \mathrm{~kg} \mathrm{CH} / 4 \mathrm{~kg} \mathrm{DBO}$ remov.) pode ser considerado conservador no sentido de superestimativa das emissões totais para estes sistemas. Medições 
diretas em reatores UASB tratando esgotos domésticos realizadas por Souza et al. (2012) revelaram emissões totais em torno de $0,38 \mathrm{~kg} \mathrm{CH}_{4} / \mathrm{kg} \mathrm{DBO}_{\text {remov. }}$ (aplicando fator de conversão 2,4 sobre o valor total contabilizado

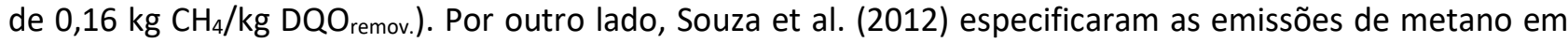
três fluxos distintos: biogás canalizado, dissolvido no efluente e superfície do decantador, com os seguintes

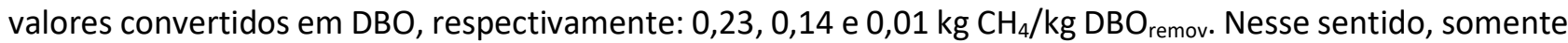
a parcela do fluxo para biogás canalizado pode ser prontamente queimada, pois as demais parcelas tendem a se constituir como emissões fugitivas sem imediato controle. Portanto, aplicando as eficiências de queima consideradas na Tabela 3 (0, 25, 50 e 95\%) somente sobre a parcela do fluxo no biogás, os fatores de emissão

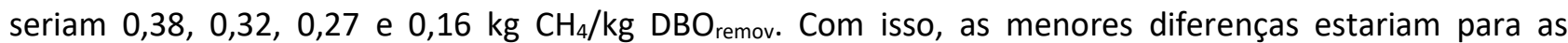
eficiências 25 e 50\%, que tiveram consideração mais determinante nos cálculos do presente estudo para as ETE de médio e pequeno porte, reforçando a validade do método de cálculo do presente estudo. Diferente do que ocorre para os reatores anaeróbios, pesquisas sobre emissões de GEE e sequestro de carbono em wetlands construídos ainda são limitadas.

\section{Considerações sobre a emissão e queima de gases nos reatores anaeróbios}

O gerenciamento das emissões gasosas em ETE empregando reatores UASB é desafiador. Como dito, apenas a parcela do biogás coletado e canalizado até os queimadores é convertida em $\mathrm{CO}_{2}$, porém, com eficiências amplamente variáveis e frequentemente inferiores a 50\%. Muitas vezes observa-se disfuncionalidade ou inoperância nestes equipamentos, seja por projeto, execução ou operação inadequados, especialmente nas ETE de pequeno porte. Por outro lado, uma relevante emissão fugitiva é o fluxo de metano dissolvido no efluente final, que é desprendido, mesmo com os queimadores operando a contento. Nesse sentido, as seguintes indicações podem representar futuras melhorias e minimização das emissões: Aprimoramentos operacionais ou de projeto, sobretudo para queimadores abertos, ou utilização de queimadores enclausurados em ETE de pequeno e médio porte (POSSETI et al, 2018; KAMINSKI et al, 2018); Aplicação de técnica específica para controlar emissões de metano e sulfeto dissolvidos em efluentes de UASB denominada câmara de dessorção. Eficiências de remoção de metano próximas de $80 \%$ foram reportadas por Souza et al. (2019) e Santos et al. (2019).

\section{Análise comparativa das estimativas de emissão de GEE nos cenários avaliados}

$\mathrm{Na}$ Erro! Fonte de referência não encontrada. apresentam-se informações que subsidiam a avaliação do impacto das alterações na matriz de tecnologias de tratamento de esgoto no Brasil por meio do incremento da participação dos wetlands construídos. Assumindo uma configuração padrão hipotética em que cada ETE por wetlands construídos atende a um equivalente de 3.000 habitantes, o número de estações utilizando estes sistemas nos cenários 01 e 02 seria, respectivamente, 816 e 2.021 ETE. Dado que, só na França - país que possui área superficial similar à do estado da Bahia - existem mais de 4.500 ETE que utilizam esta tecnologia (FRANCE, 2018), o resultado obtido demonstra ser uma perspectiva realista e, até mesmo, ainda bastante conservadora. 
Quando comparadas as estimativas de emissões anuais de metano e de gás carbônico dos cenários que consideram a participação dos wetlands construídos (01 e 02) com o cenário controle, observa-se que a redução das emissões em termos de toneladas de carbono equivalente atinge valores da ordem de 867 milhões ao ano. Esta redução, em termos do potencial de geração de receita a partir da venda de créditos de carbono, representa valores de 8 a 70 milhões de dólares ao ano (a depender do preço unitário de tonelada de carbono).

Em termos percentuais, o incremento da participação dos wetlands construídos na matriz tecnológica brasileira resultou em uma redução de 4,3\% das emissões totais provenientes do tratamento de esgotos sanitários no cenário 01 e de mais de $10 \%$ no cenário 02 . Se considerados apenas os municípios com populações iguais ou inferiores a 20.000 habitantes, onde de fato ocorreram as alterações na matriz de tecnologias, a redução das emissões representa valores da ordem de $10 \%$ (cenário 01) a 25\% (cenário 02).

Ressalta-se que, dado às suas condições demográficas, geográficas e climáticas, a utilização dos wetlands construídos no Brasil é ainda mais favorável, se comparada à realidade dos países de clima temperado, utilizados como base de referência para este estudo. Maiores percentuais de redução de emissões de GEE são possíveis em cenários mais otimistas.

Tabela 4: Emissões anuais totais de metano e de gás carbônico equivalente e análise quantitativa e monetária da redução de emissões para os cenários avaliados.

\begin{tabular}{|c|c|c|c|c|}
\hline \multirow{2}{*}{ Parâmetro analisado } & & \multicolumn{3}{|l|}{ Cenários } \\
\hline & & Controle & 01 & 02 \\
\hline \multicolumn{2}{|l|}{ Pop. atendida pelos wetlands construídos (hab) } & 0 & 2.449 .249 & 6.062 .302 \\
\hline \multicolumn{2}{|l|}{$\mathbf{N}^{\circ}$ de ETE por wetlands construídos ${ }^{(1)}$} & 0 & 816 & 2.021 \\
\hline \multicolumn{2}{|l|}{ Emissão anual $\mathrm{CH}_{4}(\mathrm{~kg} \mathrm{CH} / \mathrm{ano})$} & 290.813 .261 & 278.298 .448 & 259.852 .144 \\
\hline \multicolumn{2}{|l|}{ Emissão anual $\mathrm{CO}_{2} \mathrm{e}$ (ton $\mathrm{CO}_{2} / \mathrm{ano}$ ) } & 8.142 .771 & 7.792 .357 & 7.275 .860 \\
\hline \multicolumn{2}{|l|}{ Redução de emissões (RCE) (ton $\mathrm{CO}_{2} \mathrm{eq} / \mathrm{ano}$ ) } & - & 350.415 & 866.911 \\
\hline \multicolumn{2}{|l|}{$\begin{array}{l}\text { Redução de emissões na matriz tecnológica } \\
\text { (todas as faixas populacionais) (\%) }\end{array}$} & - & $4,3 \%$ & $10,6 \%$ \\
\hline \multicolumn{2}{|l|}{$\begin{array}{l}\text { Redução de emissões na matriz tecnológica } \\
\text { (municípios < } 20 \text { mil hab) (\%) }\end{array}$} & - & $9,9 \%$ & $24,5 \%$ \\
\hline \multirow{3}{*}{ Valor monetário crédito de carbono (USD/ano) } & Médio & - & 14.016 .591 & 34.676 .451 \\
\hline & Mínimo & - & 3.504 .148 & 8.669 .113 \\
\hline & Máximo & - & 28.033 .182 & 69.352 .902 \\
\hline
\end{tabular}

1. Considera população média de 3.000 pessoas atendida por cada ETE por wetlands construídos.

2. NOTA: Conversão de Dólar para Real à taxa de câmbio média de R\$ 5,70 em março 2021 (BCB, 2021).

\section{Considerações sobre consumo de energia e aproveitamento do biogás nas ETE}

O presente estudo não contabiliza as emissões indiretas associadas ao consumo de energia elétrica da ETE. Para pequenos/médios sistemas simplificados, este consumo pode ser considerado pouco representativo. Todavia, é importante destacar que, diferente de outras plantas de tratamento aeróbias que requerem alto consumo de energia elétrica e elevados custos com o gerenciamento do lodo produzido (ex.: lodos ativados), os sistemas wetlands construídos apresentam consumo baixo ou nulo de energia elétrica (a depender de uso ou não de aeração artificial e de bombas elétricas para alimentação), além de eliminarem a necessidade do uso de estruturas complementares para desaguamento do lodo, uma vez que este ocorre de forma passiva no próprio leito. Ainda assim, a elevada qualidade do efluente final obtida é compatível com aquela obtida em sistemas aeróbios intensivos. Por isso, diferente dos sistemas anaeróbios, os wetlands construídos não necessitam de pós tratamento. 
Já os sistemas anaeróbios que produzem o biogás canalizado, como reatores UASB, podem trazer vantagens particulares no que diz respeito ao aproveitamento do biogás. $\mathrm{O}$ aproveitamento energético, como apontado e desenvolvido por Noyola et al. (2016) para o México, pode trazer ganhos em termos de mitigação das emissões de GEE, sobretudo pensando em ETE de grande porte (maiores que 100 mil habitantes), na perspectiva de economia de energia externa da matriz elétrica tradicional. Por outro lado, segundo Souza et al. (2019), para ETE de pequeno porte, pode-se simplesmente aproveitar a energia térmica da queima do biogás, por exemplo, por meio de uso na higienização do lodo e uso doméstico nas cercanias da ETE, seja para cocção e/ou para aquecimento de água. De qualquer forma, a destruição do biogás/metano em queimadores se constitui na solução de projeto mais imediata e simples operacionalmente.

\section{Ferramenta para avaliação do benefício de implantação dos sistemas wetlands construídos sob o contexto do saneamento de baixo carbono}

Em escala local, a avaliação da redução de emissões de GEE a partir da utilização dos wetlands construídos em substituição a outras tecnologias de tratamento de esgoto torna-se relevante no âmbito de estudos de viabilidade para implantação de novas ETE que tenham como princípio o saneamento de baixo carbono.

A Tabela 5 fornece um subsídio para avaliações expeditas de comparação dos wetlands construídos com as demais tecnologias avaliadas em termos de emissões de GEE, sendo mais uma ferramenta para auxílio na tomada de decisão de gestores municipais. O cálculo da redução das emissões (toneladas de $\mathrm{CO}_{2 \text { eq }}$ que deixariam de ser emitidas com o uso dos wetlands) e dos créditos de carbono associados podem ser calculados para cada situação em função da população atendida pela ETE e da tecnologia de tratamento de esgotos alvo de comparação.

Tabela 5: Ferramenta para cálculo das reduções de emissão de GEE e obtenção de créditos de carbono com a utilização dos wetlands construídos em substituição a outras tecnologias.

\begin{tabular}{|l|l|l|}
\hline Wetlands construídos (sistema francês) em substituição a: & $\begin{array}{l}\text { Redução de emissões } \\
\text { (ton CO } \text { Creq }_{\text {hab.ano) }}\end{array}$ & Critos de carbono (USD/hab.ano) (1) \\
\hline Reatores anaeróbios sem queima de gás & 0,211 & 8,5 \\
\hline Reatores anaeróbios com 25\% de queima & 0,158 & 6,3 \\
\hline Reatores anaeróbios com 50\% de queima & 0,104 & 4,2 \\
\hline Reatores anaeróbios com 95\% de queima & 0,007 & 0,3 \\
\hline Lagoas - sistema australiano & 0,095 & 3,8 \\
\hline Tanque séptico + Filtro anaeróbio & 0,150 & 6,0 \\
\hline
\end{tabular}

1. Considera preço unitário médio do crédito de carbono de USD 40,00.

Como exemplo, apresenta-se o raciocínio do cálculo para uma ETE de 5.000 habitantes, cujo objetivo é avaliar a redução das emissões de GEE e o respectivo valor em créditos de carbono com o uso dos wetlands construídos em comparação com os reatores anaeróbios sem queima de gás. Multiplicando os valores descritos na Tabela 5 (primeira linha) pela população atendida pela ETE, conclui-se que a utilização dos wetlands construídos resulta em uma redução de emissões de 1.055 ton $\mathrm{CO}_{2 \text { eq }} /$ ano $\left(0,211\right.$ ton $\mathrm{CO}_{2 \text { eq }} /$ hab.ano X 5.000 hab), o que corresponde a um valor médio de créditos de carbono de USD 42.500,00/ano (8,5 USD/hab.ano X $5.000 \mathrm{hab})$. 


\section{Considerações sobre a viabilidade técnica e econômica de implantação dos wetlands construídos na realidade nacional}

Além dos benefícios associados à redução das emissões de GEE, a utilização dos wetlands construídos no tratamento de esgotos em municípios de pequeno e médio porte apresenta vantagens relacionadas ao baixo custo de operação e manutenção (OPEX) e à simplicidade das rotinas operacionais. A eliminação da necessidade de manejo frequente do lodo e a possibilidade de destinação agrícola do composto final gerado após ciclos operacionais de até 10 anos, equalizam o problema de muitos municípios que não possuem aterro sanitário próximo e/ou que possuem custos elevados com a logística de destinação do lodo.

O requisito de área para implantação dos wetlands construídos é considerado um fator limitante para o uso da tecnologia, uma vez que este é superior quando comparado com outras tecnologias mais compactas. Entretanto, em municípios de menor porte a disponibilidade de área em geral não representa um obstáculo. As lagoas de estabilização, por exemplo, que são os sistemas mais empregados no Brasil para estas faixas populacionais, também ocupam grandes áreas, muitas vezes superiores às que os wetlands construídos ocupariam. Cabe ressaltar que os mais recentes avanços relacionados à aplicação dos wetlands construídos demonstram ser possível reduzir as áreas necessárias em países de clima tropical para até 0,8 $\mathrm{m}^{2} /$ hab, incluindo a possibilidade de saturação do fundo dos leitos e a utilização de apenas duas unidades no primeiro estágio do sistema francês (LATUNE et al., 2017; MORAES, 2019).

Na última década, a aplicação dos wetlands construídos para pequenos municípios e aglomerados urbanos está sendo encorajada por meio de várias ações conjuntas de pesquisa e aplicação (BRASIL, 2015; TREIN et al., 2015; SCHROEDER et al., 2019; SANTOS, 2019). Mais recentemente, a Fundação Nacional de Saúde - FUNASA, vinculada ao Ministério da Saúde, apresentou à sociedade brasileira o Programa Nacional de Saneamento Rural, de onde consolida-se a aplicação de wetlands construídos para as áreas censitárias denominadas áreas urbanas isoladas, bem como para a zona rural como um todo, ampliando assim o conjunto de opções tecnológicas aplicáveis aos municípios ao longo de todo o território nacional, tonandose mais uma opção à universalização da dimensão esgotamento sanitário (BRASIL, 2019).

\section{CONCLUSÕES}

O presente estudo demonstrou que, além das vantagens técnicas e econômicas, a inserção dos wetlands construídos na matriz de tecnologias de tratamento de esgotos sanitários para municípios de até 20 mil habitantes pode trazer impactos ambientais e financeiros relevantes sob o contexto da transição para uma economia de baixo carbono.

As lagoas de estabilização, reatores anaeróbios e sistemas de tanques sépticos, principais tecnologias de tratamento de esgoto utilizadas no Brasil para essa faixa populacional, possuem fatores de emissão de metano até 80 vezes superiores aos wetlands construídos. No cenário de universalização dos serviços de saneamento, a incorporação dos wetlands construídos na matriz de tecnologias de saneamento no Brasil, considerando percentuais de participação ainda conservadores, poderia reduzir as emissões totais de metano provenientes do tratamento de esgotos sanitários em mais de $10 \%$, valor que representa cerca de 
867 milhões de toneladas de gás carbônico equivalente que deixam de ser emitidas anualmente. Se transformadas em créditos de carbono, esta redução representa uma receita média de cerca de 35 milhões de dólares ao ano, equivalente a cerca de 200 milhões de reais (BBC, 2021).

De maneira simplificada, o presente estudo fornece subsídios para o cálculo expedito das reduções de emissão de GEE e créditos de carbono associados com a utilização dos wetlands construídos em substituição a outras tecnologias de tratamento potencialmente mais poluidoras. O maior valor de redução potencial encontrado foi de 0,211 ton $\mathrm{CO}_{2 \mathrm{eq}}$ /hab.ano, quando os wetlands construídos são utilizados em substituição aos reatores anaeróbios sem queima de gás. Este constitui mais um item de comparação que pode ser levado em conta nos estudos de concepção para seleção de alternativas tecnológicas em novas ETE e/ou na busca por financiamento via programas que trabalham com o eixo de mudança climática.

\section{REFERÊNCIAS}

ANA. Agência Nacional de Águas, Secretaria Nacional de Saneamento Ambiental. Atlas esgotos: despoluição de bacias hidrográficas. Brasília: ANA, 2017a.

ANA. Atlas Esgotos: Informações por município. Brasília: ANA, 2017b.

ANA. Atlas Esgotos: Estações de Tratamento de Esgoto (2019). Brasília: ANA, 2020.

BRASIL. Ministério da Saúde, Fundação Nacional de Saúde. Manual de Saneamento. 4 ed. Brasília: Funasa, 2015.

BRASIL. Ministério da Saúde, Fundação Nacional de Saúde. Programa Nacional de Saneamento Rural. Brasília: Funasa, 2019.

BCB. Banco Central do Brasil. Relatório de Inflação. Brasília: Banco Central do Brasil, 2021.

CETESB. Primeiro Inventário Brasileiro de emissões antrópicas de gases de efeito estufa: Emissões de metano no tratamento e na disposição de resíduos. São Paulo: CETESB, 2006.

CHERNICHARO, C. A. L.; BRESSANI-RIBEIRO, T.; GARCIA, G. B.; LERMONTOV, A.; PEREIRA, C. B.; PLATZER, C. J.; POSSETTI, G. R. C.; LEITES, M. A. L.; ROSSETO, R.. Panorama do tratamento de esgoto sanitário nas regiões Sul, Sudeste e Centro-Oeste do Brasil: tecnologias mais empregadas. Revista DAE, São Paulo, v.66, n.213, p.5-19, 2018. DOI: http://doi.org/10.4322/dae.2018.028

CHERNICHARO, C. A. L.. Reatores Anaeróbios. Princípios do Tratamento Biológico de Águas Residuárias. 2 ed. Belo Horizonte: UFMG, 2016.

FRANCE. Ministère de la Transition Écologique et Solidaire. Base de Données sur les Eaux Résiduaires Urbaines (ERU) (csv). 2018.

IPCC. Climate Change 2014: Synthesis Report. Contribution of Working Groups I, II and III to the Fifth Assessment Report of the Intergovernmental Panel on Climate Change. Geneva: 2014a.
IPCC. Constructed Wetlands for Wastewater Treatment. In: 2013 Supplement to the 2006 IPCC Guidelines for National Greenhouse Gas Inventories: Wetlands. Switzerland: IPCC, 2014b.

IPCC. Wastewater Treatment and Discharge. In: 2006 IPCC Guidelines for National Greenhouse Gas Inventories: Volume 5: Waste. Japan: IGES, 2006.

IPCC. Wastewater Treatment and Discharge. In: 2019 Refinement to the 2006 IPCC Guidelines for National Greenhouse Gas Inventories: Volume 5: Waste. Switzerland: IPCC, 2019.

KADLEC, H. R.; WALLACE, S.. Treatment wetlands. 2 ed. Boca Raton: CRC Press, 2009.

LANGERGRABER, G.; WEISSENBACHER, N.. Survey on number and size distribution of treatment wetlands in Austria. Water Science and Technology, v.75, n.10, p.2309-2315, 2017.

LATUNE, R. L.; MOLLE, P.. Les filtres plantés de végétaux pour le traitement des eaux usées domestiques en milieu tropical: Guide de dimensionnement de la filière tropicalisée. France: AFB, 2017.

LIMA, A. P.; SALVADOR, N.. Geração de metano e de créditos de carbono no tratamento de esgotos sanitários. Revista DAE, São Paulo, n. 195, p.60-70, 2014. DOI: http://dx.doi.org/10.4322/dae.2014.121

MAIS, F.; BRESCIANI, R.; MARTINUZZI, N.; RIZZO, A. C. L.. Large scale application of French Reed Beds: municipal wastewater treatment for a 20,000 inhabitant's town in Moldova. Water Science and Technology, v.76, n.1, p.134146, 2017. DOI: http://doi.org/10.2166/wst.2017.188

MORAES, M. A.. Comportamento hidráulico de um sistema de wetlands construídos de escoamento vertical (primeiro estágio do sistema francês). Dissertação (Mestrado em Saneamento, Meio Ambiente e Recursos Hídricos) Universidade Federal de Minas Gerais, Belo Horizonte, 2019.

NOYOLA, A.; PAREDES, M. G.; MORGAN-SAGASTUME, J. M.; GÜERECA, L. P.. Reduction of greenhouse gas emissions from municipal wastewater treatment in Mexico based on 
technology selection. CLEAN-Soil, Air, Water, v.44, n.9, p.1091-1098, 2016. DOI: https://doi.org/10.1002/clen.201500084

SALOMON, K. R.; LORA, E. E. S.. Estimate of the electric energy generating potential for different sources of biogas in Brazil. Biomass and Bioenergy, v.33, n.9, p.1101-1107, 2009. DOI: https://doi.org/10.1016/j.biombioe.2009.03.001

SANTOS, A. B.. Caracterização, Tratamento e Gerenciamento de Subprodutos de Correntes de Esgotos Segregadas e Não Segregadas em Empreendimentos Habitacionais. Fortaleza: Imprece, 2019.

SCHROEDER, K. A.; WALZBUREICH, L.; SEZERINO, P. H.. Wetlands construídos como alternativa ao tratamento descentralizado de esgoto em Santa Catarina. In: SIMPÓSIO BRASILEIRO SOBRE WETLANDS CONSTRUÍDOS, 4. Anais. Belo Horizonte: Grupo Wetlands Brasil, 2019.

SEEG. Sistema de Estimativas de Emissões de Gases de Efeito Estufa do Observatório do Clima. Análise das emissões brasileiras de gases de efeito estufa e suas implicações para as metas do Brasil, 1970 - 2018. Brasília: SEEG, 2019.

SOUZA, C.; CHERNICHARO, C.; MELO, G.. Methane and hydrogen sulfide emissions in UASB reactors treating domestic wastewater. Water Science \& Technology, v.65, n.7, p.1229-1237, 2012.

STEFANAKIS, A.; AKRATOS, C. S.; TSIHRINTZIS, V. A.. Vertical flow constructed wetlands: eco-engineering systems for wastewater and sludge treatment. Washington: Elsevier, 2014.

STEFANAKIS, A. I.. Constructed Wetlands: description and benefits of an eco-tech water treatment system. In: Waste Management: Concepts, Methodologies, Tools, and Applications. London: IGI Global, 2020. DOI: http://doi.org/10.4018/978-1-7998-1210-4.ch025

TREIN, C. N.; PELISSARI, C.; HOFFMANN, H.; PLATZER, C. J.; SEZERINO, P. H.. Tratamento descentralizado de esgotos de empreendimentos comercial e residencial empregando a ecotecnologia dos wetlands construídos. Ambiente Construído, Porto Alegre, v.15, n.4, p.351-367, 2015. DOI: http://dx.doi.org/10.1590/s1678-86212015000400055

VON SPERLING, M.. Introdução à qualidade das águas e ao tratamento de esgotos. Princípios do Tratamento Biológico de Águas Residuárias. 4 ed. Belo Horizonte: UFMG, 2018.

VON SPERLING, M.; SEZERINO, P. H.. Dimensionamento de wetlands construídos no Brasil: Documento de consenso entre pesquisadores e praticantes. Wetlands Brasil - Grupo de estudos em sistemas wetlands construídos aplicados ao tratamento de águas residuárias. Boletim Wetlands Brasil, 2018.

WBG. State and Trends of Carbon Pricing 2019. Washington: World Bank, 2019. DOI: http://doi.org/10.1596/978-1-4648$\underline{1435-8}$

A CBPC - Companhia Brasileira de Produção Científica (CNPJ: 11.221.422/0001-03) detém os direitos materiais desta publicação. Os direitos referem-se à publicação do trabalho em qualquer parte do mundo, incluindo os direitos às renovaç̃ões, expansões e disseminações da contribuiç̃o, bem como outros direitos subsidiários. Todos os trabalhos publicados eletronicamente poderão posteriormente ser publicados em coletâneas impressas sob coordenação da Sustenere Publishing, da Companhia Brasileira de Produção Científica e seus parceiros autorizados. Os (as) autores (as) preservam os direitos autorais, mas não têm permissão para a publicação da contribuição em outro meio, impresso ou digital, em português ou em tradução. 